nephron

Practice
Nephron 2019;143:77-85

DOI: $10.1159 / 000500487$
Received: November 28, 2018

Accepted after revision: April 16, 2019

Published online: May 22, 2019

\title{
Enarodustat, Conversion and Maintenance Therapy for Anemia in Hemodialysis Patients: A Randomized, Placebo-Controlled Phase 2b Trial Followed by Long-Term Trial
}

\author{
Tadao Akizawa $^{a}$ Masaomi Nangaku ${ }^{b}$ Takuhiro Yamaguchic Masanobu Arai ${ }^{\mathrm{d}}$ \\ Ryosuke Koretomo $^{d}$ Kazuo Maeda ${ }^{d}$ Yuya Miyazawa $^{d}$ Hideki Hirakata $^{e}$ \\ ${ }^{a}$ Division of Nephrology, Department of Medicine, Showa University School of Medicine, Tokyo, Japan; ${ }^{b}$ Division of \\ Nephrology and Endocrinology, The University of Tokyo, Tokyo, Japan; ' Division of Biostatistics, Tohoku University \\ Graduate School of Medicine, Miyagi, Japan; ${ }^{d}$ Pharmaceutical Division, Japan Tobacco Inc., Tokyo, Japan; ${ }^{\mathrm{e}}$ Fukuoka \\ Renal Clinic, Fukuoka, Japan
}

\section{Keywords}

Enarodustat · Anemia in chronic kidney disease ·

Hypoxia-inducible factor prolyl hydroxylase inhibitor .

Hepcidin · Randomized trial

\begin{abstract}
Background: Enarodustat (JTZ-951) is an orally available hypoxia-inducible factor prolyl hydroxylase inhibitor that increases endogenous erythropoietin levels in the treatment of anemia associated with chronic kidney disease (CKD). Objective: A phase $2 b$ study of enarodustat to assess the hemoglobin $(\mathrm{Hb})$ response, safety, and maintenance dosage was conducted in Japanese anemic patients with hemodialysisdependent CKD. Methods: Subjects receiving a stable dose of an erythropoiesis-stimulating agent were randomized to receive once-daily enarodustat at a dose of 2,4 , or $6 \mathrm{mg}$ or
\end{abstract}

placebo in a double-blind manner for 6 weeks (Period 1) followed by 24-week open treatment with enarodustat, adjusted in the range of $2-8 \mathrm{mg}$ to maintain $\mathrm{Hb}$ within a target range (10.0-12.0 g/dL; Period 2). Results: Change in $\mathrm{Hb}$ from baseline increased with enarodustat dose in Period 1. In Period 2 , the proportion of subjects who maintained their $\mathrm{Hb}$ level within the target range at the end of treatment was $65.1 \%$. To maintain $\mathrm{Hb}$ levels within the target range over the course of Period 2, approximately $80 \%$ of subjects required 2 dose adjustments or fewer. Enarodustat decreased hepcidin and ferritin levels, increased total iron-binding capacity, and was generally well tolerated. Conclusions: Enarodustat corrected and maintained $\mathrm{Hb}$ levels in anemic patients with hemodialysis-dependent CKD. Phase 3 studies of enarodustat are currently ongoing.

(c) 2019 The Author(s) Published by S. Karger AG, Basel

\begin{tabular}{ll}
\hline KARGER & $\begin{array}{l}\text { K } 2019 \text { The Author(s) } \\
\text { Published by S. Karger AG, Basel }\end{array}$ \\
E-Mail karger@karger.com & This article is licensed under the Creative Commons Attribution- \\
www.karger.com/nef & $\begin{array}{l}\text { NonCommercial-NoDerivatives 4.0 International License (CC BY- } \\
\text { NC-ND) (http://www.karger.com/Services/OpenAccessLicense). } \\
\text { Usage and distribution for commercial purposes as well as any dis- } \\
\text { tribution of modified material requires written permission. }\end{array}$
\end{tabular}

Tadao Akizawa

Division of Nephrology, Department of Medicine

Showa University School of Medicine, Namics Shinagawa 301

4-24-51 Takanawa, Minato-ku, Tokyo 108-0074 (Japan)

E-Mail akizawa@med.showa-u.ac.jp 


\section{Introduction}

Renal anemia is a common complication in patients with chronic kidney disease (CKD). It occurs mainly as a result of decreased erythropoietin (EPO) production associated with impairment of renal function but also because of various other factors including suppressed erythropoiesis by uremic substances, shortened red blood cell survival, impaired iron metabolism, residual blood in the dialysis circuit, and malnutrition [1-4]. Several observational studies have shown a negative correlation between hemoglobin $(\mathrm{Hb})$ concentrations and mortality risk and quality of life [5-9]. However, randomized controlled studies have shown that erythropoiesis-stimulating agent (ESA) therapy with high $\mathrm{Hb}$ targets increased mortality and cardiovascular events [10-12]. Thus, the Guideline for Renal Anemia in CKD of the Japanese Society for Dialysis Therapy recommends target maintenance $\mathrm{Hb}$ levels of $10.0-12.0 \mathrm{~g} / \mathrm{dL}$ for hemodialysis patients [13].

Enarodustat (JTZ-951) is being developed as an orally available drug to promote hematopoiesis via endogenous EPO production. It acts by inhibiting the prolyl hydroxylase domain-containing protein that regulates the activity of hypoxia-inducible factors (HIFs) involved in the production of EPO. Hypoxia can lead to decreased prolyl hydroxylase activity and stabilization of HIF- $\alpha$ in the liver and kidney, resulting in increased production of endogenous EPO and hematopoiesis [14].

A phase 2a study in renal anemia patients on maintenance hemodialysis showed that 8-week therapy of enarodustat with dose titration from 1 to $5 \mathrm{mg}$ resulted in a dose-related increase in $\mathrm{Hb}$ without safety concerns [15]. In the present phase $2 \mathrm{~b}$ study in renal anemia patients on maintenance hemodialysis, we report the efficacy of enarodustat after switching from an ESA, the safety of enarodustat 2-8 mg administered for a maximum of 30 weeks, and the maintenance dose.

\section{Materials and Methods}

\section{Study Design}

This phase $2 \mathrm{~b}$ study was conducted at 16 sites from May 2015 to June 2016 and consisted of a 4-week screening period, 30-week treatment period, and 2 -week follow-up period. The treatment period consisted of 2 parts: a double-blind, randomized, placebocontrolled, parallel-arm, comparative study (Period 1) and an open-label, uncontrolled, intra-individual dose-adjustment extension study (Period 2). Patients with renal anemia on maintenance hemodialysis and treated with ESAs were included. In Period 1, subjects were randomized in a 1:1:1:1 ratio to receive once-daily oral enarodustat 2, 4, $6 \mathrm{mg}$ or placebo for 6 weeks to evaluate the dose-response relationship and safety of enarodustat. Subsequently, in Period 2, the dose of enarodustat in individual subjects was adjusted within a range of $2-8 \mathrm{mg}$ to maintain the target $\mathrm{Hb}$ level $(10.0-12.0 \mathrm{~g} / \mathrm{dL})$ for 24 weeks. In Period 2, the long-term safety and maintenance dose of enarodustat were also evaluated. The starting dose in Period 2 was either $4 \mathrm{mg}$ (for subjects with $\mathrm{Hb} \geq 8.0$ to $\leq 12.0 \mathrm{~g} / \mathrm{dL}$ at Week 6 ) or $2 \mathrm{mg}$ (for subjects with $\mathrm{Hb}>12.0$ to $<13.0 \mathrm{~g} / \mathrm{dL}$ at Week 6), with dose adjustments every 4 weeks. Study treatment was suspended when $\mathrm{Hb} \geq 13.0 \mathrm{~g} / \mathrm{dL}$. This study was registered with the Japan Pharmaceutical International Center Clinical Trials Information registry (JapicCTI-152892) in May 2015. The study was conducted in compliance with the ethical principles that have their origin in the Declaration of Helsinki; the protocol; and the Guidelines for Good Clinical Practice of Japanese Ministerial Ordinance. The study was approved by the Institutional Review Board of each study center. Written informed consent was obtained from all subjects prior to participation.

\section{Subjects and Treatment}

The Period 1 inclusion criteria were: Japanese men and women aged $\geq 20$ years; hemodialysis or hemodiafiltration 3 times per week for at least 12 weeks prior to the start of the screening period (Scr Visit 1); ESA therapy for at least 4 weeks prior to Scr Visit 1; mean $\mathrm{Hb}$ level at Scr Visit 1 and 2 weeks later (Scr Visit 2) of 9.5$12.0 \mathrm{~g} / \mathrm{dL}$ with an absolute difference of $\leq 1.0 \mathrm{~g} / \mathrm{dL}$; and transferrin saturation $>20 \%$ or ferritin $>75 \mathrm{ng} / \mathrm{mL}$ at Scr Visit 1 . The Period 1 main exclusion criteria were: onset of myocardial infarction, cerebral infarction, or venous thromboembolism in the 24 weeks prior to Scr Visit 1; intact-parathyroid hormone $\geq 500 \mathrm{pg} / \mathrm{mL}$ at Scr Visit 1 ; receipt of an erythrocyte transfusion or surgery involving massive blood loss in the 12 weeks prior to Scr Visit 1; and anemia secondary to chronic disease, systemic blood disorder, or obvious bleeding such as gastrointestinal hemorrhage. Further details are provided in the online supplementary materials (for all online suppl. material, see www.karger.com/doi/10.1159/000500487).

Subjects who completed Period 1 with $\mathrm{Hb}$ levels of $\geq 8.0$ to $<13.0 \mathrm{~g} / \mathrm{dL}$ at Week 6 were eligible for inclusion in Period 2.

Intravenous iron use was prohibited during the screening period and during Period 1. Oral iron therapy initiated before Scr Visit 1 was permitted to be continued without any change in dose. In Period 2, iron supplementation was permitted only if necessary.

\section{Assessments}

The primary endpoint in Period 1 was the percentage of subjects with a change in the $\mathrm{Hb}$ level within $\pm 1.0 \mathrm{~g} / \mathrm{dL}$ from baseline to the evaluation-point, which was defined as the average of the last 2 measurements in Period 1 (i.e., end of Period 1 treatment and one time point earlier), while baseline was defined as the average of the 3 pre-therapy measurements obtained at Scr Visit 1, Scr Visit 2, and Week 0.

In Period 2, efficacy was evaluated based on the time course of $\mathrm{Hb}$ level, target $\mathrm{Hb}(\geq 10.0$ to $\leq 12.0 \mathrm{~g} / \mathrm{dL})$ maintenance rate, mean prescribed dose, and number of dose adjustments. No primary endpoint was established in Period 2.

Safety was evaluated based on adverse events (AEs) occurring after the administration of study drug, laboratory tests, vital signs, standard 12-lead electrocardiogram, chest X-ray, and fundoscopy findings.

In addition, iron-related parameters were examined as an exploratory endpoint. 
Statistical Analysis

All analyses were performed using SAS software version 9.3 or higher (SAS Institute, Cary, NC, USA).

Based on our previous study [15], for the determination of the sample size, it was assumed that the proportion of subjects maintaining an $\mathrm{Hb}$ level within the range $\pm 1.0 \mathrm{~g} / \mathrm{dL}$ from baseline would be $70 \%$ in the enarodustat arm and $10 \%$ in the placebo arm. With a $2.5 \%$ one-sided significance level and $90 \%$ power using Fisher's exact test, the required number of subjects per arm was calculated to be 16 . To allow for possible withdrawals and dropouts, the target sample size per arm was set to 20 . The Period 1 efficacy full analysis set (FAS) consisted of subjects who received study drug and who underwent efficacy evaluation on 2 or more occasions. The Period 2 FAS consisted of subjects who proceeded to Period 2 and who underwent efficacy evaluation on one or more occasions. The safety population consisted of subjects who received study drug and underwent safety-related assessments on one or more occasions.

The primary endpoint was evaluated by comparing the results from the placebo arm as a control with each of the enarodustat 2 , 4, and $6 \mathrm{mg}$ arms using Fisher's exact test (significance level, 2.5\% one-sided). A permutation test was used for multiplicity adjustment. In addition, post hoc analysis of the primary endpoint (i.e., the percentage of subjects achieving a change in the $\mathrm{Hb}$ level within $\pm 1.0 \mathrm{~g} / \mathrm{dL}$ from baseline to evaluation-point) was performed using Fisher's exact test to verify the superiority of enarodustat to placebo. Iron-related parameters were assessed in the safety population. A post hoc analysis of the changes in iron-related parameters at Week 6 was performed using a Wilcoxon rank-sum test for inter-arm comparison between the placebo arm and each of the enarodustat dose arms (significance level, 5\% two-sided). As this was an exploratory study, no multiplicity was taken into account.

AEs occurring from the start of study drug administration to the follow-up observation 2 weeks after the last dose of the study drug were summarized. All AEs were coded using the Medical Dictionary for Regulatory Activities/Japanese, version 18.0.

\section{Results}

\section{Subject Disposition and Characteristics}

In Period 1, 85 subjects were randomized to placebo or to one of the 3 enarodustat arms. Of these, 82 were included in the FAS after the exclusion of 3 subjects ( 2 in the $2 \mathrm{mg}$ arm and 1 in the $6 \mathrm{mg}$ arm) with $<2$ efficacy measurements. All 85 subjects received study drug and were included in the safety population. Period 1 was completed by 68 subjects, of whom 63 subjects proceeded to Period 2 , which was completed by 55 subjects (Fig. 1). Subject baseline characteristics in each arm are shown in Table 1.

\section{Primary Endpoint (Period 1)}

The mean baseline $\mathrm{Hb}$ level in each arm was $10.54 \pm$ $0.64 \mathrm{~g} / \mathrm{dL}$ in the placebo arm, $10.39 \pm 0.50 \mathrm{~g} / \mathrm{dL}$ in the enarodustat $2 \mathrm{mg}$ arm, $10.59 \pm 0.65 \mathrm{~g} / \mathrm{dL}$ in the enarodustat $4 \mathrm{mg}$ arm, and $10.48 \pm 0.60 \mathrm{~g} / \mathrm{dL}$ in the enarodustat
$6 \mathrm{mg}$ arm. In the placebo arm, the percentage of subjects with a change in the $\mathrm{Hb}$ level within $\pm 1.0 \mathrm{~g} / \mathrm{dL}$ from baseline to evaluation-point was $27.3 \%$. By contrast, this percentage was $63.2 \%$ in the $2 \mathrm{mg}$ arm, $60.0 \%$ in the $4 \mathrm{mg}$ arm, and $52.4 \%$ in the $6 \mathrm{mg}$ arm, and tended to be higher than in the placebo arm without a significant difference ( $p=0.0311$ for the $2 \mathrm{mg}$ arm, $p=0.0457$ for the $4 \mathrm{mg}$ arm, and $p=0.1189$ for the $6 \mathrm{mg}$ arm). A post hoc analysis using Fisher's exact test demonstrated that the percentage of subjects who achieved a change in the $\mathrm{Hb} \mathrm{lev-}$ el within $\pm 1.0 \mathrm{~g} / \mathrm{dL}$ from baseline to evaluation-point was significantly higher in the enarodustat arm than in the placebo arm $(p=0.0118)$.

The specific change in the $\mathrm{Hb}$ level from baseline to evaluation-point in each arm was $-1.27 \mathrm{~g} / \mathrm{dL}$ (95\% CI -1.68 to $-0.87 \mathrm{~g} / \mathrm{dL})$ in the placebo arm, $-0.62 \mathrm{~g} / \mathrm{dL}(95 \%$ CI -1.15 to $-0.09 \mathrm{~g} / \mathrm{dL})$ in the $2 \mathrm{mg}$ arm, $0.38 \mathrm{~g} / \mathrm{dL}(95 \%$ CI -0.10 to $0.85 \mathrm{~g} / \mathrm{dL}$ ) in the $4 \mathrm{mg}$ arm, and $0.89 \mathrm{~g} / \mathrm{dL}$ (95\% CI $0.47-1.31 \mathrm{~g} / \mathrm{dL})$ in the $6 \mathrm{mg}$ arm, showing greater changes in the $\mathrm{Hb}$ level at higher doses of enarodustat. The $4 \mathrm{mg}$ arm demonstrated no significant change in the $\mathrm{Hb}$ level after switching from ESAs to enarodustat.

\section{Maintenance of $\mathrm{Hb}$ (Period 2)}

Twenty-four-week enarodustat therapy in reference to protocol-specified dose adjustments led to appropriate control of $\mathrm{Hb}$ levels within the target range of 10.0-12.0 g/dL (Fig. 2). In subjects who proceeded to Period 2, the mean $\mathrm{Hb}$ level was $10.14 \mathrm{~g} / \mathrm{dL}$ (95\% CI 9.85-10.43 g/dL) at Week 6 (prior to study drug administration in Period 2), $10.63 \mathrm{~g} / \mathrm{dL}$ (95\% CI 10.38-10.88 g/dL) at Ext Week 24 (24 weeks after the start of study drug administration in Period 2), and $10.49 \mathrm{~g} / \mathrm{dL}$ (95\% CI $10.24-10.74 \mathrm{~g} / \mathrm{dL}$ ) at the end of treatment. The target $\mathrm{Hb}$ maintenance rate at Week 6 was 52.4\%, which increased to $70.9 \%$ at Ext Week 24 and $65.1 \%$ at the end of treatment.

In subjects assigned in the placebo arm in Period 1, enarodustat administration also led to increased $\mathrm{Hb} \mathrm{lev-}$ els in Period 2. Specifically, at Week 6, the mean Hb level was $9.26 \mathrm{~g} / \mathrm{dL}$ (95\% CI 8.84-9.67 g/dL) and the target $\mathrm{Hb}$ maintenance rate was $25.0 \%$, both of which increased to $10.63 \mathrm{~g} / \mathrm{dL}$ (95\% CI 10.05-11.20 g/dL) and 64.3\%, respectively, at Ext Week 8.

In subjects who proceeded to Period 2, the mean prescribed dose of study drug in Period 2 was $4.30 \mathrm{mg} /$ day. The study drug compliance rate in Period 2 was $97.9 \%$. The number of required dose adjustments was zero (i.e., the dose was not changed from the starting dose for Period 2 ) in $27.0 \%$ of subjects, 1 or 2 in $52.4 \%$, and 3 or more in $20.6 \%$. 


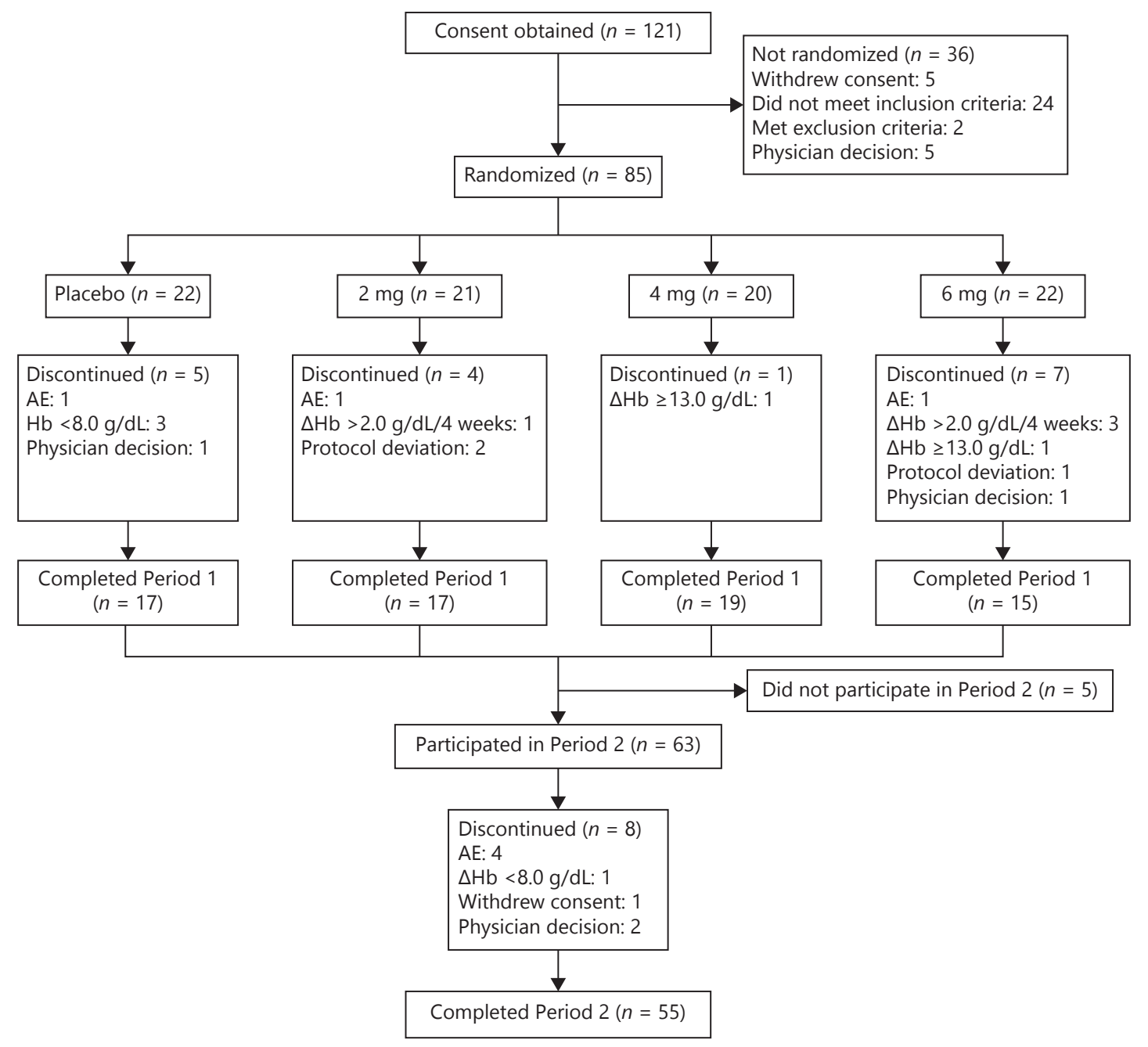

Fig. 1. Subject disposition. Hb, hemoglobin; AE, adverse event.

\section{Iron-Related Parameters}

The changes in iron-related parameters over time in Period 1 are shown in Figure 3. The iron-related parameters at Week 0 are shown in online supplementary Table S1. For hepcidin and ferritin, the changes from Week 0 tended to be decreased in the enarodustat arms compared with the placebo arm, and were significant in the enarodustat 4 and $6 \mathrm{mg}$ arms compared with the placebo arm. For total iron-binding capacity, the changes from Week 0 were significantly increased in all enarodustat arms compared with the placebo arm. These iron-related parameters remained stable over time in Period 2. In subjects who were in the placebo arm of Period 1, administration of enarodustat in Period 2 led to an increase in $\mathrm{Hb}$ level, and in parallel, the iron-related parameters showed similar changes to those in the enarodustat arms of Period 1 .

Intravenous iron preparations were used at least once during Period 2 in 16 (25.4\%) of the 63 subjects, with a mean $( \pm$ SD) total dose of $430.0 \pm 227.9 \mathrm{mg}$. Oral iron preparations were used during Period 2 in $5(7.9 \%)$ of the 63 subjects.

\section{Safety}

No deaths occurred during this study. In Period 1, AEs occurred in 31 of 63 subjects in the enarodustat arm (49.2\%) and 14 of 22 subjects in the placebo arm (63.6\%). No AEs were observed to have a higher incidence at higher doses of enarodustat. Regarding serious adverse events (SAEs) in Period 1, basal ganglia infarction occurred in 
Fig. 2. Hb levels over time. The plot represents the mean $\mathrm{Hb}$ level of each treatment arm for each day of observation. Bars indicate SD. Period 1: Week 0-6, Period 2: Week 6-Ext Week 24. Hb, hemoglobin.

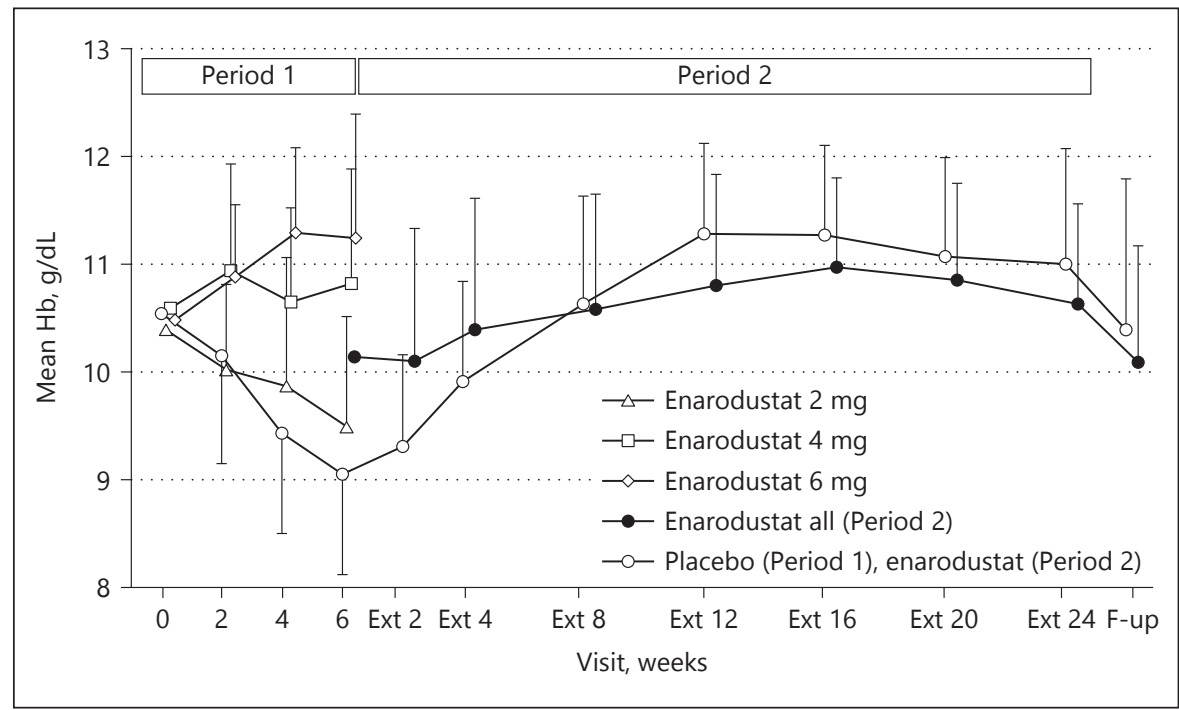

Table 1. Subject characteristics

\begin{tabular}{|c|c|c|c|c|c|}
\hline Characteristic & $\begin{array}{l}\text { Placebo } \\
(n=22)\end{array}$ & $\begin{array}{l}\text { Enarodustat } \\
2 \mathrm{mg}(n=19)\end{array}$ & $\begin{array}{l}\text { Enarodustat } \\
4 \mathrm{mg}(n=20)\end{array}$ & $\begin{array}{l}\text { Enarodustat } \\
6 \mathrm{mg}(n=21)\end{array}$ & $\begin{array}{l}\text { Total } \\
(n=82)\end{array}$ \\
\hline Age, years, mean (SD) & $60.7(13.0)$ & $60.1(7.7)$ & $66.1(9.3)$ & $60.8(13.0)$ & $61.9(11.2)$ \\
\hline \multicolumn{6}{|l|}{ Gender, $n(\%)$} \\
\hline Male & $15(68.2)$ & $15(78.9)$ & $14(70.0)$ & $16(76.2)$ & $60(73.2)$ \\
\hline Female & $7(31.8)$ & $4(21.1)$ & $6(30.0)$ & $5(23.8)$ & $22(26.8)$ \\
\hline Body weight, kg, mean (SD) & $58.1(11.6)$ & $65.1(13.3)$ & $58.8(11.1)$ & $62.0(11.7)$ & $60.9(12.0)$ \\
\hline \multicolumn{6}{|l|}{ Primary disease of CKD, $n(\%)$} \\
\hline Chronic glomerulonephritis & $6(27.3)$ & $11(57.9)$ & $12(60.0)$ & $8(38.1)$ & $37(45.1)$ \\
\hline Diabetic nephropathy & $2(9.1)$ & $4(21.1)$ & $6(30.0)$ & $7(33.3)$ & $19(23.2)$ \\
\hline Nephrosclerosis & $6(27.3)$ & $2(10.5)$ & $0(0.0)$ & $4(19.0)$ & $12(14.6)$ \\
\hline Other & $8(36.4)$ & $2(10.5)$ & $2(10.0)$ & $2(9.5)$ & $14(17.1)$ \\
\hline \multicolumn{6}{|l|}{ Prior ESA, $n(\%)$} \\
\hline rHuEPO & $10(45.5)$ & $10(52.6)$ & $9(45.0)$ & $10(47.6)$ & $39(47.6)$ \\
\hline Darbepoetin alfa & $10(45.5)$ & $9(47.4)$ & $10(50.0)$ & $8(38.1)$ & $37(45.1)$ \\
\hline Epoetin beta pegol & $2(9.1)$ & $0(0.0)$ & $1(5.0)$ & $3(14.3)$ & $6(7.3)$ \\
\hline \multicolumn{6}{|l|}{ Prior ESA dose, mean (SD) } \\
\hline rHuEPO, IU/week & $3,075.0(1,296.6)$ & $4,575.0(2,014.0)$ & $4,166.7(2,681.0)$ & $2,250.0(1,620.2)$ & $3,500.0(2,088.5)$ \\
\hline Darbepoetin alfa, $\mu \mathrm{g} /$ week & $13.3(5.0)$ & $13.1(10.4)$ & $11.5(5.3)$ & $16.6(11.9)$ & $13.4(8.3)$ \\
\hline Epoetin beta pegol, $\mu \mathrm{g} / 4$ weeks & 75.0 & N/A & 100.0 & $150.0(132.3)$ & $116.7(93.1)$ \\
\hline Oral iron, $n(\%)$ & $2(9.1)$ & $1(5.3)$ & $0(0.0)$ & $0(0.0)$ & $3(3.7)$ \\
\hline
\end{tabular}

For body weight and eGFR, data at Scr Visit 1 are shown. For oral iron, the number of users at Scr Visit 1 is shown.

eGFR, estimated glomerular filtration rate; CKD, chronic kidney disease; ESA, erythropoiesis-stimulating agent; rHuEPO, recombinant human erythropoietin; N/A, not applicable.

one subject in the enarodustat $6 \mathrm{mg}$ arm, and after discontinuation of the study drug, this subject had a retinal artery occlusion as an SAE. For both SAEs, a causal relationship to the study drug was ruled out by the investigator. In 2 other subjects (one each in the $2 \mathrm{mg}$ arm and the placebo arm), AEs (nausea in 1 subject in the $2 \mathrm{mg}$ arm, cerebral infarction in 1 subject in the placebo arm) led to the discontinuation of study drug.

AEs occurring in $\geq 5 \%$ of subjects receiving enarodustat as well as all SAEs occurring after administration of enarodustat are summarized in Table 2. In Period 2, SAEs were noted in 6 subjects, including coronary artery steno- 


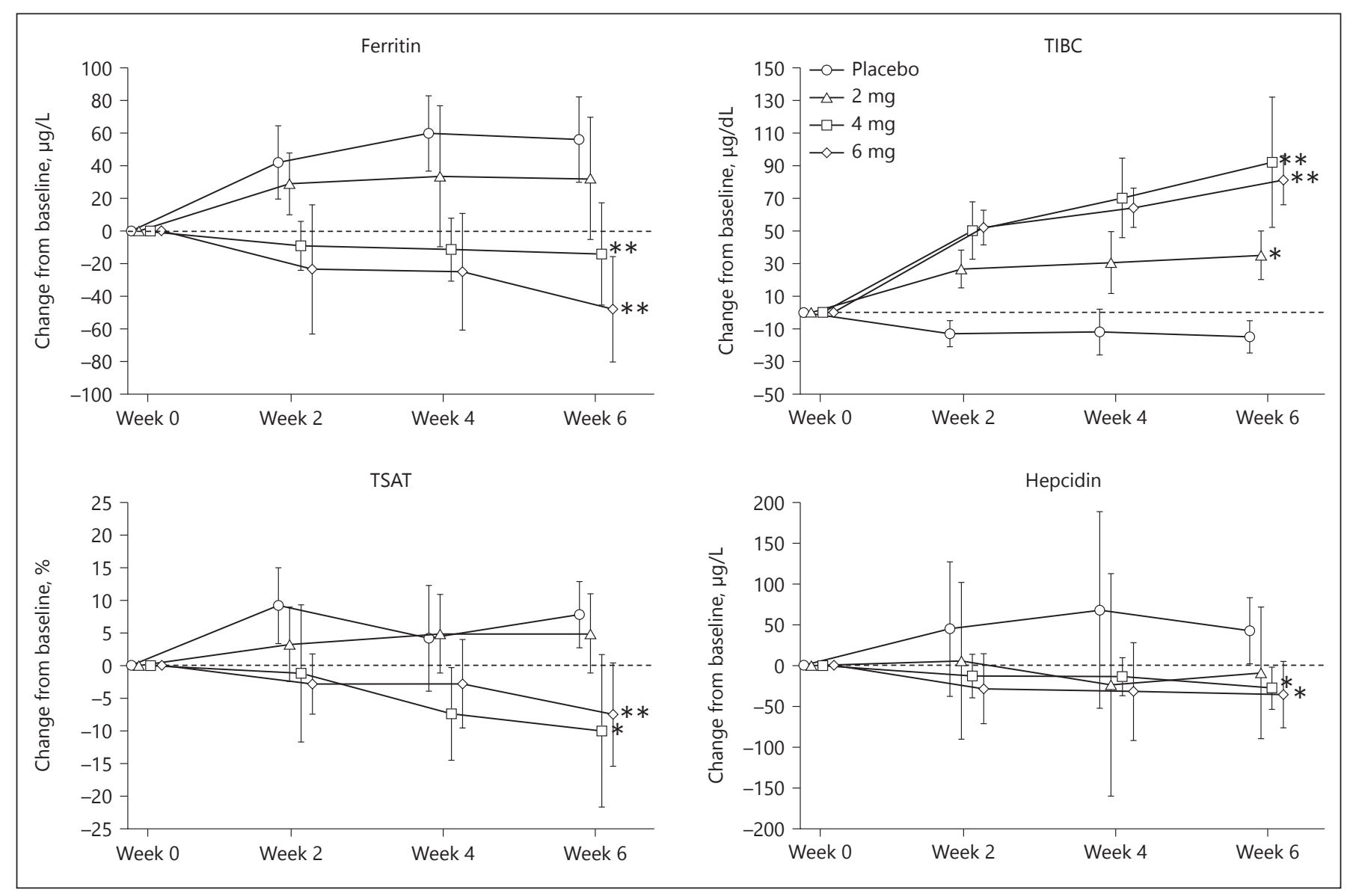

Fig. 3. Changes in iron-related parameters (Period 1). Each plot represents the median change of each treatment arm for each day of observation, and the vertical bar on each plot represents quartile deviation. A post hoc analysis was performed on the change at Week 6 using the Wilcoxon rank-sum test for inter-arm compari-

sis in one subject, for which a causal relationship to the study drug was not ruled out by the investigator. In Period 2, AEs led to the discontinuation of study drug in 4 subjects, but none of these AEs occurred in more than 1 subject who withdrew because of AEs.

Laboratory tests, vascular endothelial growth factor (VEGF), EPO, vital signs, standard 12-lead electrocardiogram, chest X-ray, and fundoscopy showed no obvious changes throughout the study period.

\section{Discussion/Conclusion}

Enarodustat is an orally available HIF-PH inhibitor under development for the treatment of anemia associated with CKD. The Period 1 results of this study showed

son between the placebo arm and each of the enarodustat arms (significance level, 5\% two-sided). No multiplicity was taken into account. ${ }^{*} p<0.05,{ }^{* *} p<0.0001$. BL, baseline; TIBC, total ironbinding capacity; TSAT, transferrin saturation.

that $\mathrm{Hb}$ levels in renal anemia patients on regular hemodialysis were maintained without abrupt changes after switching from an ESAs to enarodustat. In the enarodustat $4 \mathrm{mg}$ arm, no significant change in the $\mathrm{Hb}$ level was observed from baseline to evaluation-point, suggesting that ESA therapy can be appropriately switched to enarodustat. In addition, the Period 2 results indicated stable $\mathrm{Hb}$ control within the target range in $70 \%$ or more of subjects from Ext Week 12 onward following enarodustat dose adjustment within the range of 2-8 $\mathrm{mg}$ based on $\mathrm{Hb}$ level, including subjects who were in the placebo arm in Period 1. In Period 2, the number of required dose adjustments was $\leq 2$ in almost $80 \%$ of subjects.

As shown in Figure 2, the Hb level at the Ext F-up Visit (2 weeks after Ext Week 24) was lower than at Ext 
Table 2. AEs reported in $5 \%$ or more subjects and any SAEs

\begin{tabular}{ll}
\hline & $\begin{array}{c}\text { Enarodustat total } \\
(n=79), n(\%)\end{array}$ \\
\hline AEs ( $\geq 5 \%$ subjects) & \\
Any AEs & $66(83.5)$ \\
Nasopharyngitis & $31(39.2)$ \\
Constipation & $7(8.9)$ \\
Upper respiratory tract infection & $7(8.9)$ \\
Diarrhea & $5(6.3)$ \\
Excoriation & $5(6.3)$ \\
Oropharyngeal pain & $5(6.3)$ \\
Pruritus & $5(6.3)$ \\
Contusion & $4(5.1)$ \\
Shunt stenosis & $4(5.1)$ \\
Wound & $4(5.1)$ \\
Arthralgia & $4(5.1)$ \\
SAEs & $2(2.5)$ \\
Pneumonia & $1(1.3)^{\mathrm{a}}$ \\
Retinal artery occlusion & $1(1.3)$ \\
Procedural hypertension & $1(1.3)$ \\
Tonsillitis & $1(1.3)^{\mathrm{c}}$ \\
Colon cancer & $1(1.3)^{\mathrm{a}, \mathrm{c}}$ \\
Basal ganglia infarction & $1(1.3)^{\mathrm{b}}$ \\
Coronary artery stenosis & $1(1.3)$ \\
Ventricular tachycardia & $1(1.3)$ \\
Myelopathy & $1(1.3)$ \\
Vertigo positional & $1(1.3)^{\mathrm{c}}$ \\
Subdural hematoma & $1(1.3)^{\mathrm{c}}$ \\
Spinal cord injury cervical & $1(1.3)^{\mathrm{c}}$ \\
Cerebral hematoma & \\
\hline a SAEs in Period 1. & \\
b Assessed as causally related to the study & \\
gator. by the investi- & \\
c SAE requiring discontinuation of study drug. \\
AEs, adverse events; SAEs, serious adverse events. \\
\hline
\end{tabular}

Week 24, indicating attenuation of the effect of enarodustat in maintaining the $\mathrm{Hb}$ level within 2 weeks. This suggests that, even when $\mathrm{Hb}$ exceeds the upper limit of the target range, it can be promptly lowered by treatment suspension.

Conventional ESAs need to be administered at doses above the range of physiological fluctuations of EPO [16]. In patients with ESA hypo-responsiveness, higher doses of ESAs are required to increase $\mathrm{Hb}$ levels. However, high-dose ESA therapy has been reported to increase the risks of cardiovascular events and mortality [17]. Furthermore, hemodialysis patients on ESA often cannot efficiently utilize iron in the body, resulting in a state of relative iron deficiency, and thus require intravenous iron administration despite the potential risk of ad- verse effects of iron such as infections and cardiovascular risk [18-24]. Enarodustat may overcome these limitations via 2 mechanisms. First, enarodustat increases $\mathrm{Hb}$ levels without excessive EPO production above the physiological range. In a study of repeated dosing of enarodustat for 15 days in patients on hemodialysis, $\mathrm{Hb}$ levels increased along with physiological EPO levels. Furthermore, no accumulation of EPO was observed after repeated dosing of enarodustat [25]. Similarly, in this study, EPO levels in placebo-treated subjects in Period 1 were marginally increased in response to enarodustat treatment (online suppl. Table S2), and remained within the physiological range during Period 2. Second, the mechanism by which iron-related parameters are modulated by enarodustat treatment may differ from that associated with ESA treatment. The enarodustat arms in Period 1 showed decreased hepcidin compared with the placebo arm. HIF regulates the expression of genes encoding hepcidin, and HIF stabilization has been reported to suppress hepcidin [26]. Although the mechanism by which hepcidin is suppressed by an ESA through erythroferrone production has previously been reported [2729], a trend toward a decrease in hepcidin was observed in this study following switching from an ESA to enarodustat. The effect of enarodustat in improving iron metabolism and iron supplementation needs to be further investigated in a phase 3 study using an ESA as a comparator.

Regarding safety, no AEs occurring more frequently at higher doses of enarodustat, and no AEs were observed that were characteristic of enarodustat compared with other HIF-PH inhibitors [30-32]. AEs related to hypertension occurred in 3 subjects during enarodustat therapy, but all 3 subjects had a complication of hypertension. No increase in mean systolic or diastolic blood pressure was observed during treatment in the safety population (data not shown).

HIF is also known to regulate the expression of VEGF, and stabilization of HIF may lead to increased expression of VEGF [33]. Increased VEGF expression is implicated in the aggravation of diabetic retinopathy, cancer, and other diseases featuring angiogenesis [34-35]. Similar to other studies on HIF-PH inhibitors [30-32], enarodustat administration in this study did not cause changes in VEGF (online suppl. Table S2). However, this finding requires further investigation using safety data from a phase 3 study with ESA as a comparator and in a long-term study.

In conclusion, the administration of enarodustat switched from an ESA did not cause abrupt changes in $\mathrm{Hb}$ 
levels in patients with renal anemia on maintenance hemodialysis, and subsequent dose adjustments led to appropriate control of $\mathrm{Hb}$ levels. The present study raised no major safety concerns for phase 3 study implementation. Currently, a phase 3 study is ongoing to evaluate the long-term safety and efficacy of enarodustat, and a study using an ESA as a comparator is also expected to support the efficacy and safety of enarodustat.

\section{Acknowledgment}

We would like to thank the physicians, nurses, and patients at the participating centers for their support. We also acknowledge the support of ASCA Corporation (http://www.asca-co.com/english_site/) for editing a draft of this manuscript.

\section{Statement of Ethics}

All patients provided written informed consent prior to participation. The study was registered with the Japan Pharmaceutical International Center Clinical Trials Information (JapicCTI-152892) and was conducted in compliance with the ethical principles of the Declaration of Helsinki; the protocol; and the
Guidelines for Good Clinical Practice of the Japanese Ministerial Ordinance, and was approved by the Institutional Review Board at each participating study site.

\section{Disclosure Statement}

T.A., T.Y., and H.H.: are consultants and received consulting fees from Japan Tobacco Inc. M.N.: is a consultant and has received grants and personal fees from Japan Tobacco Inc. M.A., R.K., K.M., and Y.M.: are employees and have stock or stock options in Japan Tobacco Inc.

\section{Funding Sources}

Funding for this research was provided by Japan Tobacco Inc.

\section{Author Contributions}

All authors contributed to the final analysis and interpretation of data, and had full access to the study data and analyses. All authors contributed to revising the article, providing intellectual content of clinical importance to the work described, and final approval of the version to be published.

\section{References}

1 Artunc F, Risler T. Serum erythropoietin concentrations and responses to anaemia in patients with or without chronic kidney disease. Nephrol Dial Transplant. 2007 Oct;22(10): 2900-8.

2 Eschbach JW, Adamson JW. Anemia of endstage renal disease (ESRD). Kidney Int. 1985 Jul;28(1):1-5.

3 Vos FE, Schollum JB, Coulter CV, Doyle TC, Duffull SB, Walker RJ. Red blood cell survival in long-term dialysis patients. Am J Kidney Dis. 2011 Oct;58(4):591-8.

4 Babitt JL, Lin HY. Molecular mechanisms of hepcidin regulation: implications for the anemia of CKD. Am J Kidney Dis. 2010 Apr; 55(4):726-41.

5 Ofsthun N, Labrecque J, Lacson E, Keen M, Lazarus JM. The effects of higher hemoglobin levels on mortality and hospitalization in hemodialysis patients. Kidney Int. 2003 May; 63(5):1908-14.

6 Regidor DL, Kopple JD, Kovesdy CP, Kilpatrick RD, McAllister CJ, Aronovitz J, et al. Associations between changes in hemoglobin and administered erythropoiesis-stimulating agent and survival in hemodialysis patients. J Am Soc Nephrol. 2006 Apr;17(4):1181-91.

7 Akizawa T, Pisoni RL, Akiba T, Saito A, Fukuhara S, Asano Y, et al. Japanese haemodialysis anaemia management practices and outcomes
(1999-2006): results from the DOPPS. Nephrol Dial Transplant. 2008 Nov;23(11):3643-53.

8 Inaba $\mathrm{M}$, Hayashino $\mathrm{Y}$, Shoji T, Akiba T, Akizawa T, Saito A, et al. Disappearance of association in diabetic patients on hemodialysis between anemia and mortality risk: the Japan dialysis outcomes and practice pattern study. Nephron Clin Pract. 2012;120(2):c91-100.

9 Akizawa T, Saito A, Gejyo F, Suzuki M, Nishizawa Y, Tomino Y, et al.; JET Study Group. Low hemoglobin levels and hypo-responsiveness to erythropoiesis-stimulating agent associated with poor survival in incident Japanese hemodialysis patients. Ther Apher Dial. 2014 Oct;18(5):404-13.

10 Parfrey PS, Foley RN, Wittreich BH, Sullivan DJ, Zagari MJ, Frei D. Double-blind comparison of full and partial anemia correction in incident hemodialysis patients without symptomatic heart disease. J Am Soc Nephrol. 2005 Jul;16(7):2180-9.

11 Besarab A, Bolton WK, Browne JK, Egrie JC, Nissenson AR, Okamoto DM, et al. The effects of normal as compared with low hematocrit values in patients with cardiac disease who are receiving hemodialysis and epoetin. N Engl J Med. 1998 Aug;339(9):584-90.

12 Palmer SC, Navaneethan SD, Craig JC, Johnson DW, Tonelli M, Garg AX, et al. Metaanalysis: erythropoiesis-stimulating agents in patients with chronic kidney disease. Ann Intern Med. 2010 Jul;153(1):23-33.

13 Yamamoto H, Nishi S, Tomo T, Masakane I, Saito K, Nangaku M, et al. 2015 Japanese society for dialysis therapy: guidelines for renal anemia in chronic kidney disease. Ren Replace Ther. 2017 Jun;3(1):36.

14 Takeda K, Aguila HL, Parikh NS, Li X, Lamothe K, Duan LJ, et al. Regulation of adult erythropoiesis by prolyl hydroxylase domain proteins. Blood. 2008 Mar;111(6):3229-35.

15 Akizawa T, Hanaki K, Arai M. JTZ-951, An oral novel HIF-PHD inhibitor, elevates hemoglobin in Japanese anemic patients with chronic kidney disease receiving maintenance hemodialysis. Nephrol Dial Transplant. 2015 May;30(suppl 3):iii10.

16 Jelkmann W. The ESA scenario gets complex: from biosimilar epoetins to activin traps. Nephrol Dial Transplant. 2015 Apr;30(4): 553-9.

17 McCullough PA, Barnhart HX, Inrig JK, Reddan D, Sapp S, Patel UD, et al. Cardiovascular toxicity of epoetin-alfa in patients with chronic kidney disease. Am J Nephrol. 2013;37(6): 549-58.

18 Agarwal R, Kusek JW, Pappas MK. A randomized trial of intravenous and oral iron in chronic kidney disease. Kidney Int. 2015 Oct; 88(4):905-14. 
19 Litton E, Xiao J, Ho KM. Safety and efficacy of intravenous iron therapy in reducing requirement for allogeneic blood transfusion: systematic review and meta-analysis of randomised clinical trials. BMJ. 2013 Aug 15; 347:f4822.

20 Brookhart MA, Freburger JK, Ellis AR, Wang L, Winkelmayer WC, Kshirsagar AV. Infection risk with bolus versus maintenance iron supplementation in hemodialysis patients. J Am Soc Nephrol. 2013 Jun;24(7):1151-8.

21 Kuo KL, Hung SC, Lin YP, Tang CF, Lee TS, Lin CP, et al. Intravenous ferric chloride hexahydrate supplementation induced endothelial dysfunction and increased cardiovascular risk among hemodialysis patients. PLoS One. 2012;7(12):e50295.

22 Reis KA, Guz G, Ozdemir H, Erten Y, Atalay $\mathrm{V}$, Bicik Z, et al. Intravenous iron therapy as a possible risk factor for atherosclerosis in endstage renal disease. Int Heart J. 2005 Mar; 46(2):255-64.

23 Drüeke T, Witko-Sarsat V, Massy Z, Descamps-Latscha B, Guerin AP, Marchais SJ, et al. Iron therapy, advanced oxidation protein products, and carotid artery intima-media thickness in end-stage renal disease. Circulation. 2002 Oct; 106(17):2212-7.

24 Akizawa T, Okumura H, Alexandre AF, Fukushima A, Kiyabu G, Dorey J. Burden of ane- mia in chronic kidney disease patients in Japan: a literature review. Ther Apher Dial. 2018 Oct;22(5):444-56.

25 Pai S, Koretomo R, Tamaki S, Berg J, Marbury T, Galloway C, et al. JTZ-951, a novel HIFPHD inhibitor, demonstrates increases in hemoglobin, iron mobilization, reproducible pharmacokinetics, and safety following once daily administration for 15 days in patients with anemia receiving hemodialysis. Nephrol Dial Transplant. 2015 May;30(Suppl 3): iii293-4.

26 Peyssonnaux C, Nizet V, Johnson RS. Role of the hypoxia inducible factors HIF in iron metabolism. Cell Cycle. 2008 Jan;7(1):28-32.

27 Kautz L, Jung G, Valore EV, Rivella S, Nemeth E, Ganz T. Identification of erythroferrone as an erythroid regulator of iron metabolism. Nat Genet. 2014 Jul;46(7):678-84.

28 Gammella E, Diaz V, Recalcati S, Buratti P, Samaja M, Dey S, et al. Erythropoietin's inhibiting impact on hepcidin expression occurs indirectly. Am J Physiol Regul Integr Comp Physiol. 2015 Feb;308(4):R330-5.

29 Ganz T, Jung G, Naeim A, Ginzburg Y, Pakbaz Z, Walter PB, et al. Immunoassay for human serum erythroferrone. Blood. 2017 Sep; 130(10):1243-6.

30 Akizawa T, Tsubakihara Y, Nangaku M, Endo Y, Nakajima H, Kohno T, et al. Effects of daprodustat, a novel hypoxia-inducible factor prolyl hydroxylase inhibitor on anemia management in Japanese hemodialysis subjects. Am J Nephrol. 2017;45(2):127-35.

31 Provenzano R, Besarab A, Wright S, Dua S, Zeig S, Nguyen P, et al.Roxadustat (FG-4592) Versus Epoetin Alfa for Anemia in Patients Receiving Maintenance Hemodialysis: A Phase 2, Randomized, 6- to 19-Week, OpenLabel, Active-Comparator, Dose-Ranging, Safety and Exploratory Efficacy Study. Am J Kidney Dis. 2016 Jun;67(6):912-24.

32 Haase VH, Chertow GM, Block GA, Pergola PE, deGoma EM, Khawaja Z, et al. Effects of vadadustat on hemoglobin concentrations in patients receiving hemodialysis previously treated with erythropoiesis-stimulating agents. Nephrol Dial Transplant. 2019 Jan 1;34(1):90-

33 Liu Y, Cox SR, Morita T, Kourembanas S. Hypoxia regulates vascular endothelial growth factor gene expression in endothelial cells. Identification of a 5' enhancer. Circ Res. 1995 Sep;77(3):638-43.

34 Goel HL, Mercurio AM. VEGF targets the tumour cell. Nat Rev Cancer. 2013 Dec;13(12): $871-82$

35 Abcouwer SF. Angiogenic factors and cytokines in diabetic retinopathy. J Clin Cell Immunol. 2013;1(11 Suppl 1) 